\title{
A integralidade do cuidado na saúde do homem: um enfoque na qualidade de vida
}

\author{
The comprehensive care in men's health: an approach on quality of life
}

\section{La integralidad de la atención en la salud del hombre: un enfoque en la calidad de vida}

\author{
Gabriela Romano Oliveira. Faculdade de Medicina de Marília (FAMEMA). Marília, SP, Brasil. gabifamema@yahoo.com.br (Autora correspondente) \\ Matheus Luis Castelan Trilico. Faculdade de Medicina de Marilia (FAMEMA). Marília, SP, Brasil. matheus.trilico@gmail.com \\ Felipe Sanches Paro. Faculdade de Medicina de Marília (FAMEMA). Marília, SP, Brasil. felipeparo@hotmail.com \\ Marinei Yuko Kijimura. Faculdade de Medicina de Marília (FAMEMA). Marília, SP, Brasil. mari_kijimura@hotmail.com \\ Sueli Moreira Pirolo. Faculdade de Medicina de Marília (FAMEMA). Marília, SP, Brasil. pirolo@terra.com.br
}

\section{Resumo}

Objetivo: analisar o conceito e a percepção dos homens sobre qualidade de vida. Métodos: empregou-se o método de pesquisa descritivo-exploratória com abordagem qualitativa, realizada com homens, moradores adstritos a uma unidade da Estratégia Saúde da Família do município de Marília, São Paulo, integrada ao Programa de Educação pelo Trabalho para a Saúde (PET-Saúde). Foi empregada a técnica de construção de Discursos do Sujeito Coletivo utilizando as seguintes perguntas norteadoras: 0 que é qualidade de vida? e Como está sua qualidade de vida? Resultados: os discursos analisados evidenciaram que a maioria dos homens não compreende o sentido amplo do conceito qualidade de vida, fundamentando-o predominantemente na dimensão biológica. Conclusão: a política da saúde do homem constitui importante estratégia de promoção da saúde, todavia requer agilidade e criatividade dos profissionais da atenção primária para lidarem com a singularidade masculina e suas vulnerabilidades.

\section{Abstract}

Objective: To analyze men's concepts and perceptions about quality of life. Methods: A descriptive and exploratory qualitative study in which we interviewed male dwellers assisted by the Family Health Strategy in a health center in the municipality of Marília, São Paulo state, integrated to the "Programa de Educação pelo Trabalho para a Saúde (PET-Saúde)". The Collective Subject Discourse construction technique was applied guided by the following questions: "What is quality of life?" and "How is your quality of life?" Results: The discourses analyzed showed that most men do not understand the broad sense of the quality of life concept, substantiating it predominantly in its biological dimensions. Conclusion: Men's health policy is an important strategy for health promotion; however, it requires agility and creativity by primary healthcare staff to deal with men's uniqueness and vulnerabilities.

\section{Resumen}

Objetivo: analizar el concepto y la percepción de los hombres acerca de la calidad de vida. Métodos: se utilizó el método de investigación descriptivo exploratorio, de enfoque cualitativo, con el que fueron entrevistados hombres vinculados a la Estrategia de Salud Familiar de un centro de salud en Marília, São Paulo, integrado al Programa de Educação pelo Trabalho para a Saúde (PET-Saúde). Se utilizó la técnica de construcción de Discursos del Sujeto Colectivo usando las siguientes preguntas orientadoras: “¿Qué es calidad de vida?” y “Cómo está su calidad de vida?" Resultados: Ios discursos analizados mostraron que la mayoría de los hombres no entienden el significado amplio del concepto calidad de vida, basándolo principalmente en su dimensión biológica. Conclusión: la política de la salud del hombre es una importante estrategia de promoción de la salud que, sin embargo, requiere la agilidad y creatividad de los profesionales de atención primaria para hacer frente a las singularidades y vulnerabilidades de los hombres.

\section{Palavras-chave:}

Qualidade de Vida Saúde do Homem Homens Atenção Primária à Saúde

Keywords:

Quality of Life Men's Health Men Primary Health Care

Palabras clave: Calidad de Vida Salud del Hombre Hombres Atención Primaria de Salud 


\section{Introdução}

Ao longo das duas últimas décadas, os homens têm sido pauta de debates no campo da saúde coletiva, tanto em âmbito nacional como internacional. Isso se deve ao crescente aumento nos índices de morbimortalidade, à aparente relutância dos homens em procurar auxílio e à suposta relação antagônica com o cuidado, o que exige das políticas públicas de saúde e, por conseguinte, dos profissionais de saúde uma nova postura e outro olhar ${ }^{1-4}$.

MacDonald 5 assinala, como fatores que impedem o desenvolvimento de uma política de saúde eficaz para os homens, a preocupaçáo limitada com a perspectiva biomédica (ênfase na próstata e disfunção erétil) e o senso comum de que todos os problemas de saúde em homens são os resultados de masculinidade hegemônica e mau comportamento, sem levar em conta a percepção do indivíduo do sexo masculino com relação à sua vida e sua saúde.

Contudo, a Política Nacional de Atenção Integral à Saúde do Homem aponta para uma perspectiva de buscar qualidade de vida e promoção da integralidade do cuidado na populaçáo masculina. A Organizaçáo Mundial de Saúde (OMS) define qualidade de vida como a "[...] percepção do indivíduo de sua proteção na vida, no contexto da cultura e sistema de valores nos quais ele vive e em relação aos seus objetivos, expectativas, padrôes e preocupações.” Identifica-se nessa definição uma natureza multifatorial da qualidade de vida que considera seis domínios que objetivam demonstrar diferentes dimensóes do ser humano, as quais contemplam saúde física, saúde psicológica, nível de independência, relaçôes sociais, meio ambiente e padrão espiritual ${ }^{6,7}$.

A integralidade do cuidado na atenção à saúde deve ser pautada em políticas e açóes programáticas que respondam às demandas e necessidades da populaçáo masculina no acesso à rede de cuidados em saúde, considerando a complexidade e as especificidades de diferentes abordagens do cuidado no processo saúde-doença em suas distintas dimensôes: biológica, cultural e social ${ }^{8}$.

Deve-se entender a promoção de qualidade de vida nos homens como uma lógica de trabalho pautada em uma reorganização das práticas e, assim, as equipes transdisciplinares de saúde deveriam estar qualificadas para proporcionar consciência em saúde, colaborando com a busca de uma sociedade comprometida com seu próprio bem-estar?.

O objetivo do presente estudo foi analisar o conceito e a percepção dos homens sobre qualidade de vida, tomando como base a área de abrangência de uma unidade de saúde da família com vistas a buscar sua representação de consciência em saúde, a qual repercute no seu próprio bem-estar.

\section{Métodos}

Trata-se de uma pesquisa descritiva e exploratória com abordagem qualitativa, realizada no período de janeiro a novembro de 2011. Os participantes receberam esclarecimentos a respeito do objetivo e método, da garantia do sigilo e anonimato, da livre opção em participar ou cancelar sua permissão, sem penalidade alguma. A pesquisa foi realizada em uma unidade de saúde da Estratégia Saúde da Família (ESF) inserida no Programa de Educaçáo pelo Trabalho em Saúde, PET-Saúde.

O universo estatístico existente foi de 608 homens (30\% da população geral), os quais foram convidados a comparecer na ESF para conversarem a respeito da saúde do homem, em dias previamente agendados, nos períodos noturno e diurno. Para a composição da amostra, foram utilizados os seguintes critérios de inclusão: ter emprego e moradia fixos e idade entre 19 e 65 anos. Tais critérios foram eleitos considerando-se a realidade sociodemográfica da área de abrangência da ESF referência para essa população, tendo sido incluídos todos os homens que aceitaram participar da pesquisa. Foram excluídos os que não preencheram os critérios de inclusão ou aqueles que se recusaram a participar da pesquisa.

A técnica de amostragem utilizada foi a não probabilística ${ }^{10}$, por acessibilidade e, para a coleta de dados, foi elaborado um roteiro de entrevista semiestruturada, com perguntas abertas referentes à identificaçáo, como o homem conceitua a qualidade de vida e como ele a percebe. O teste-piloto foi realizado para adequaçáo de termos.

Para a análise dos dados, utilizou-se o Discurso do Sujeito Coletivo, técnica metodológica que permite o resgate de discursos recuperando o pensamento e a concepçáo sobre qualidade de vida, enquanto comportamento discursivo e fato social internalizado individualmente de forma qualitativa ${ }^{10}$. 
As categorias foram elaboradas ao serem agrupadas ideias semelhantes, sentidos confluentes oriundos de leituras exaustivas dos depoimentos. Identificou-se então quatro categorias de discursos, elaboradas com base na natureza multidimensional do conceito de qualidade de $\operatorname{vida}^{11}$ : (A) física - percepção do indivíduo sobre sua condição física; (B) psicológica - percepção do indivíduo sobre sua condiçáo afetiva e cognitiva; (C) do relacionamento social - percepção do indivíduo sobre os relacionamentos sociais e os papéis sociais adotados na vida; (D) do ambiente - percepçáo do indivíduo sobre aspectos diversos relacionados ao ambiente em que vive.

O presente estudo foi aprovado pelo Comitê de Ética em Pesquisa da Faculdade de Medicina de Marília (FAMEMA) sob protocolo no $1214 / 10$.

\section{Resultados}

Foram entrevistados 57 participantes na faixa etária entre 19 e 65 anos. Quanto ao perfil sociodemográfico, predominaram homens com segundo grau completo e com renda proporcional até três salários mínimos (Tabela 1).

Os dados qualitativos explicam a categorização da ideia central e a síntese do discurso do sujeito coletivo, bem como a frequência das ideias na categorização (Tabelas 2 e 3).

Tabela 1. Perfil sociodemográfico dos homens participantes da pesquisa, vinculados a uma unidade da Estratégia Saúde da Família em Marília, 2011.

\begin{tabular}{|c|c|c|}
\hline Perfil dos entrevistados $(n=57)$ & $\mathrm{N}$ & $\%$ \\
\hline \multicolumn{3}{|l|}{ Faixa etária, $\mathrm{n}(\%)$} \\
\hline $19-28$ anos & 11 & $(19,30)$ \\
\hline 20-37 anos & 13 & $(22,80)$ \\
\hline 38-46 anos & 16 & $(28,07)$ \\
\hline 47-55 anos & 13 & $(22,80)$ \\
\hline+55 anos & 04 & $(7,02)$ \\
\hline \multicolumn{3}{|l|}{ Escolaridade, $n(\%)$} \\
\hline Primeiro grau completo & 12 & $(21,05)$ \\
\hline Primeiro grau incompleto & 08 & $(14,03)$ \\
\hline Segundo grau completo & 21 & $(36,84)$ \\
\hline Segundo grau incompleto & 05 & $(8,77)$ \\
\hline Superior completo & 08 & $(14,03)$ \\
\hline Superior incompleto & 03 & $(5,26)$ \\
\hline \multicolumn{3}{|l|}{ Renda mensal, $\mathrm{n}(\%)$} \\
\hline Mais de 6 salários mínimos & 04 & $(7,02)$ \\
\hline 3-6 salários mínimos & 20 & $(35,00)$ \\
\hline Até 3 salários mínimos & 33 & $(57,89)$ \\
\hline Não Declararam & 10 & $(17,54)$ \\
\hline
\end{tabular}


Tabela 2. Dados referentes ao conceito de qualidade de vida dos homens participantes da pesquisa, vinculados a uma unidade da Estratégia Saúde da Família em Marília, 2011.

\begin{tabular}{|c|c|c|}
\hline \multicolumn{3}{|r|}{ Pergunta: 0 que é qualidade de vida para você? } \\
\hline Dimensão & $\%$ & Discurso do Sujeito Coletivo (DSC) desta dimensão \\
\hline & & $\begin{array}{l}\text { Para mim, qualidade de vida consiste em não sofrer um colapso, um acidente, estar saudável podendo cuidar da saúde para } \\
\text { trabalhar além de cuidar da minha vida, vivendo em paz e valorizando-a. }\end{array}$ \\
\hline A & 45 & $\begin{array}{l}\text { É ter uma boa alimentação e disposição, ter um bom sono e acordar cedo, praticar exercícios físicos como caminhada. É não } \\
\text { tomar bebida alcoólica, não fumar, não ter nenhum vício e ter uma boa higiene pessoal. } \\
\text { É procurar sempre um médico e tomar o remédio certo. }\end{array}$ \\
\hline $\mathrm{C}$ & 6,67 & $\begin{array}{l}\text { Qualidade de vida é estar bem com a sociedade e em paz com todos, tendo um bom relacionamento com a família, com os } \\
\text { amigos, no trabalho e com os vizinhos. }\end{array}$ \\
\hline D & 6,67 & $\begin{array}{l}\text { Qualidade de vida é curtir a vida com saúde que impulsiona a buscar os sonhos, se sentindo bem com o que faz no trabalho e } \\
\text { na vida. Não ter nenhum problema, ter saúde para trabalhar e ganhar dinheiro e estar sem dificuldades financeiras, sem dever } \\
\text { nada para ninguém. É estar bem estruturado, não deixando faltar nada em casa e poder dar aos filhos o que eles necessitam. }\end{array}$ \\
\hline
\end{tabular}

Tabela 3. Dados referentes à percepção de qualidade de vida pelos homens participantes da pesquisa, vinculados a uma unidade da Estratégia Saúde da Família em Marília, 2011.

\begin{tabular}{|c|c|c|}
\hline \multicolumn{3}{|r|}{ Pergunta: Como está a sua qualidade de vida? } \\
\hline Dimensão & $\%$ & Discurso do Sujeito Coletivo (DSC) desta dimensão \\
\hline A & 35,71 & $\begin{array}{l}\text { Está boa, pois me alimento bem e procuro sempre estar com saúde, sempre exercitando o corpo, praticando esporte, como } \\
\text { caminhada. Eu busco estar sem o colesterol e triglicérides altos. Acho que ainda precisa melhorar, pois como pão com } \\
\text { mortadela, salsicha e batata frita. }\end{array}$ \\
\hline B & 17,86 & $\begin{array}{l}\text { Não está boa por causa da rotina da vida que é muito corrida. Ando estressado, indo da casa para o trabalho e do trabalho para } \\
\text { casa. Tento fazer as coisas certas, mas não consigo, pois a correria do dia a dia faz o tempo voar. } \\
\text { Queria poder tirar férias ao invés de vendê-las para pagar as contas. }\end{array}$ \\
\hline C & 28,57 & $\begin{array}{l}\text { Não está muito boa, está regular. Tenho muita preocupação com os filhos e falta tempo para me entender com eles. As coisas } \\
\text { em casa estão passando por vários desentendimentos com muita cobrança da mulher e dos amigos. Mas o lado bom é que } \\
\text { tenho alguém para amar. }\end{array}$ \\
\hline D & 17,86 & $\begin{array}{l}\text { Está boa, pois tenho minha casa, meus filhos estão trabalhando e eu também. Vou levando a vida de pobre, mas dá pra } \\
\text { sobreviver. }\end{array}$ \\
\hline
\end{tabular}

\section{Discussão}

Os depoimentos apontam que os homens desconhecem o sentido amplo do conceito qualidade de vida, focando predominantemente em aspectos físicos e biológicos. Isso evidencia que há uma fragilidade na sensibilização dos homens para a importância das medidas de prevenção e manutenção da saúde, o que leva à dificuldade de adaptarem-se à hábitos de vida saudáveis. Torna-se necessário investir em educação para valorizar açôes que evitem comportamentos de risco à saúde pelos homens ${ }^{12}$.

Educar vem do latim educare e significa "[...] auxiliar a desenvolver o ser humano de dentro para fora." No entanto, os homens possuem um imaginário masculino de considerarem-se fortes e invulneráveis, que os impede de praticarem ações saudáveis ${ }^{12}$.

Gomes ${ }^{13}$ chama esse imaginário de marcas identitárias da masculinidade que podem fazer com que os homens se exponham mais frequentemente a fatores de risco, em muitas situaçóes tornando-se vítimas de seus próprios estilos de vida.

Em função disso, é necessário que os profissionais de saúde adotem formas diferentes de pensar e rompam com atitudes, crenças e valores cristalizados ao longo de sua formaçáo profissional e social, incorporando novos conceitos pertencentes à saúde do homem, para, consequentemente, agirem de maneira distinta em sua prática profissional, melhor organizando os serviços e estimulando estes homens para que busquem qualidade de vida ${ }^{11,14}$. 
A promoção de discussóes no âmbito da saúde pública, quanto aos sentidos atribuídos à sexualidade masculina, necessita ultrapassar as dimensôes simbólicas do imaginário masculino para que possam ser implementadas medidas preventivas e práticas de promoção da saúde com vistas a se alcançar uma integralidade do cuidado ${ }^{15}$.

\section{Conclusão}

O presente estudo teve o propósito de apresentar o conceito e analisar a percepção dos homens acerca da qualidade vida, com vistas a buscar sua representaçáo de consciência em saúde, utilizando um enfoque abrangente das principais questôes teórico-metodológicas que caracterizam sua aplicação no campo da saúde.

O desafio que se coloca para os pesquisadores brasileiros no que se refere à saúde do homem é compreender a visão fragmentada do processo do adoecimento e cuidado. Existem dois paradoxos colocados nessas agendas: como o homem lida com a sua própria saúde e cuida de si e de que maneira as políticas públicas e o sistema de saúde se organizam e compreendem a realidade masculina.

Portanto, a política da saúde do homem constitui importante estratégia de promoção da saúde, todavia requer agilidade e criatividade dos profissionais de saúde para lidarem com a singularidade masculina e suas vulnerabilidades no cotidiano dos serviços da atenção primária.

\section{Referências}

1. Carrara S, Russo JA, Faro L. A política de atenção à saúde do homem no Brasil: os paradoxos da medicalização do corpo masculino. Physis. 2009; 19(3): 659-78.

2. Pinheiro RS, Viacava F, Travassos C, Brito SA. Gênero, morbidade, acesso e utilização de serviços de saúde no Brasil. Ciênc Saúde Coletiva. 2002; 7(4): 687-707. http://dx.doi.org/10.1590/S1413-81232002000400007

3. Couto MT, Pinheiro TF, Valença O, Machin R, Silva GSN, Gomes R, et al. O homem na atenção primária à saúde: discutindo (in)visibilidade a partir da perspectiva de gênero. Interface Comun Saúde Educ. 2010; 14(33): 257-70.

4. Schraiber LB. Equidade de gênero e saúde: o cotidiano das práticas no Programa Saúde da Família do Recife. In: Villela W, Monteiro S, editores. Gênero e saúde: Programa Saúde da Família em questão. Rio de Janeiro: Abrasco; 2005. p. 39-61

5. Macdonald, JJ. Shifting paradigms: a social-determinants approach to solving problems in mens's health policy and practice. MJA. 2006 Oct [cited 2004 Maio]; 185(8). Disponível em: http://www.mja.com.au/public/issues/185_08_161006/mac10104_fm.html

6. Seidl EMF, Zannon CMLC. Qualidade de vida e saúde: aspectos conceituais e metodológicos. Cad Saúde Pública. 2004; $20: 580-8$.

7. Bub MB, Medrano C, Silva CD, et al. A noção de cuidado de si mesmo e o conceito de autocuidado na enfermagem. Texto Contexto Enferm. 2006;15: 152-7. http://dx.doi.org/10.1590/S0104-07072006000500018

8. Cecílio LCO. As necessidades de saúde como conceito estruturante na luta pela integralidade e equidade na atenção em saúde. In: Pinheiro R, Mattos RA, editores. Os sentidos da integralidade na atenção e no cuidado à saúde. Rio de Janeiro: Ed. UERJ; 2001. p. 113-26.

9. Buss PM. Promoção da saúde e qualidade de vida. Ciênc Saúde Coletiva. 2000; 5(1): 163-77. http://dx.doi.org/10.1590/S1413-81232000000100014

10. Lefevre F, Lefevre AM. O sujeito coletivo que fala. Interface Comun Saúde Educ. 2006; 10(20): 517-24. http://dx.doi.org/10.1590/S141432832006000200017

11. Minayo MCS, Hartz ZMA, Buss PM. Qualidade de vida e saúde: um debate necessário. Ciênc Saúde Coletiva. 2000; 5(1): 7-18. http://dx.doi. org/10.1590/S1413-81232000000100002

12. Schraiber LB, Gomes R, Couto MT. Homens na pauta da saúde coletiva. Ciênc Saúde Coletiva. 2005; 10: 7-17. http://dx.doi.org/10.1590/S141381232005000100002

13. Gomes R. Sexualidade masculina e saúde do homem: proposta para uma discussão. Ciênc Saúde Coletiva. 2003; 8(3): 825-9. http://dx.doi. org/10.1590/S1413-81232003000300017

14. Wang ML, Jablonski B, Magalhães. As identidades masculinas: limites e probabilidades. Psicol Rev. 2006; 12(19): 54-65.

15. Carvalho SR. As contradições da promoção à saúde em relação à produção de sujeitos e a mudança social. Ciênc Saúde Coletiva. 2004; 9(3): 669-78. http://dx.doi.org/10.1590/S1413-81232004000300018 